\title{
Article \\ A Pilot Evaluation of mHealth App Accessibility for Three Top-Rated Weight Management Apps by People with Disabilities
}

\author{
Erin Radcliffe ${ }^{1, *}$, Ben Lippincott ${ }^{1}$, Raeda Anderson ${ }^{1,2}{ }^{\oplus}$ and Mike Jones ${ }^{1}$ \\ 1 Shepherd Center, Atlanta, GA 30309, USA; ben.lippincott@shepherd.org (B.L.); \\ raeda.anderson@shepherd.org (R.A.); mike.jones@shepherd.org (M.J.) \\ 2 Department of Sociology, Georgia State University, Atlanta, GA 30302, USA \\ * Correspondence: erin.radcliffe@shepherd.org
}

Citation: Radcliffe, E.; Lippincott, B.; Anderson, R.; Jones, M. A Pilot Evaluation of mHealth App Accessibility for Three Top-Rated Weight Management Apps by People with Disabilities. Int. J. Environ. Res. Public Health 2021, 18, 3669. https:// doi.org/10.3390/ijerph18073669

Academic Editor: Paul B. Tchounwou

Received: 5 March 2021

Accepted: 30 March 2021

Published: 1 April 2021

Publisher's Note: MDPI stays neutral with regard to jurisdictional claims in published maps and institutional affiliations.

Copyright: (c) 2021 by the authors. Licensee MDPI, Basel, Switzerland. This article is an open access article distributed under the terms and conditions of the Creative Commons Attribution (CC BY) license (https:// creativecommons.org/licenses/by/ $4.0 /)$.

\begin{abstract}
Growing evidence demonstrates that people with disabilities face more challenges in accessing healthcare and wellness resources, compared to non-disabled populations. As mobile applications focused on health and wellness (mHealth apps) become prevalent, it is important that people with disabilities can access and use mHealth apps. At present, there is no source of unified information about the accessibility and usability of mHealth apps for people with disabilities. We set out to create such a source, establishing a systematic approach for evaluating app accessibility. Our goal was to develop a simple, replicable app evaluation process to generate useful information for people with disabilities (to aid suitable app selection) and app developers (to improve app accessibility and usability). We collected data using two existing assessment instruments to test three top-rated weight management apps with nine users representing three disability groups: vision, dexterity, and cognitive impairment. Participants with visual impairments reported the lowest accessibility ratings, most challenges, and least tolerance for issues. Participants with dexterity impairments experienced significant accessibility-related difficulties. Participants with cognitive impairments experienced mild difficulties and higher tolerances for issues. Our pilot protocol will be applied to test mHealth apps and populate a "curation" website to assist consumers in selecting mHealth apps.
\end{abstract}

Keywords: mHealth; mobile applications; accessibility; usability; evaluation methods; user needs discovery; inclusive design

\section{Introduction}

Over $80 \%$ of people with disabilities in the US live with chronic conditions that compound the effects of disability on health and function. There is growing evidence that people with various types of disabilities face more challenges in accessing healthcare, as well as preventive health and wellness management resources [1-3]. Mobile health (mHealth) is emerging as an important tool for managing chronic health conditions, but people with disabilities have not been a primary target for mHealth app development, and accessibility needs are often overlooked or disregarded [4]. Common accessibility needs for people with dexterity or visual impairments include app compatibility with alternative access software and assistive devices $[5,6]$. People with visual and cognitive impairments often need to use magnification software, custom contrast, color adjustments, and auditory alerts to access their mobile devices and apps [7,8]. Rapid proliferation of mHealth apps could further increase health disparities between people with and without disabilities if apps disproportionately benefit non-disabled populations. Early evidence suggests that disparities in adopting mHealth apps among people with disabilities may already exist $[9,10]$. While many popular, commercially available "diet and exercise" apps have been evaluated for efficacy and general ease of use, there is limited information 
available about accessibility and usability by people with disabilities [11-15]. Given this scarcity of information, we set out to develop a model for evaluating mHealth apps for accessibility. We conducted a concurrent mixed methods usability and accessibility evaluation of three top-rated, commercially available mHealth apps that assist users in reaching diet, exercise/fitness, and weight management goals.

Our objectives for this study were to develop and test a prototype app accessibility testing protocol and to disseminate findings via an app curation website that provides information to people with disabilities about mHealth app suitability, based on their needs, impairments, and mobile device access methods. Our findings are also intended to inform recommendations to mobile app developers for how to resolve common accessibility issues and fulfill inclusive design requirements.

\section{Materials and Methods}

\section{1. mHealth App Selection Process}

We identified the three top-rated, commercially available weight management apps, based on subject-matter-expert recommendations $[15,16]$ and comprehensive reviews evaluating popular mHealth apps for general usability and effectiveness $[13,14,17,18]$. The three apps were: MyFitnessPal, Lose It!, and FatSecret [19-21]. We chose these three apps based on the following selection criteria:

1. High consumer review ratings evenly reported on both the iOS App Store and in Google Play

2. High download/installation user-base on both iOS and Android OS devices

3. Suggested by subject matter experts in content evaluation methodology for commercial mobile health apps supporting healthy eating and physical activity $[15,16]$

4. Identified by published research reviewing smartphone apps aimed towards promoting healthy nutrition [13], physical activity [14,17], and weight management [18]

5. Commercially available on both iOS and Android OS devices

6. Free with in-app purchases for both iOS and Android device users

Ten mobile apps met the minimum thresholds for consideration: MyFitnessPal, Lose It!, Noom [22], FatSecret, WW (Weight Watchers) [23], MyPlate [24], VA MOVE! Coach [25], MyNetDiary [26], My Diet Diary [27], and SparkPeople [28]. We chose the following three apps, as they best satisfied our selection criteria:

MyFitnessPal is a weight management app that focuses on personalized diet and exercise tracking [19]. Two efficacy trials found that MyFitnessPal, compared with standard interventions for dietary self-monitoring and weight management, was equivalent in effectiveness and offered reliable compatibility with commercial hardware (e.g., Fitbit and Garmin activity trackers) to support participant diet and fitness goals [29,30].

Lose It! is a weight-loss app that supports goal-setting and nutrient tracking [20]. In a study evaluating data captured in Lose It!, results indicate successful weight loss outcomes in distinct subgroups and identify characteristics among subgroups who experienced more success in reaching their goals [31].

FatSecret is a calorie-counter app that enables food and exercise logging paired with a peer support network to reinforce users' weight-loss goals [21]. A comparison study evaluating standard versus mHealth app supported self-monitoring demonstrates preliminary evidence suggesting that diet and fitness tracking using FatSecret may produce more successful weight management outcomes [32].

\subsection{App Accessibility Testing Protocol}

Our app accessibility testing protocol was informed by a review of prior research regarding mHealth app evaluation methods, rapid user-testing methods, and a standardized system for quantifying usability feedback [33-35]. We evaluated accessibility and usability issues by asking participants to complete the following tasks within each of the three apps:

1. Explore: initial familiarization to app screens, functions, and navigational elements

2. Core Tasks: logging meals, physical activity, and diet or fitness goals 
This approach allowed us to assess general ease of use, application compatibility with built-in and external accessibility aids, and the impact of accessibility issues on each user's ability to perform primary tasks within each app. We used standardized evaluation methods from two existing measures of software usability and accessibility-the System Usability Scale (SUS) [35] and Tierney's 7-min Accessibility Assessment and App Rating System [34].

\subsubsection{System Usability Scale (SUS)}

The SUS is a standardized measure providing a quantifiable assessment of subjective usability assessments [36,37]. The SUS can provide reliable results with small sample sizes [38,39]. In multi-survey studies, the total SUS score, with a maximum of 100 , averages at 69.69 with a range of 30.00-93.93 [38].

After completing each task in our app assessment, participants were asked to score the following 10 items on a 5-point Likert scale ranging from "strongly disagree" (1) to "strongly agree" (5):

1. I think that I would like to use this system frequently.

2. I found the system unnecessarily complex.

3. I thought the system was easy to use.

4. I think I would need the support of a technical person to be able to use this system.

5. I found the various functions in this system were well integrated.

6. I thought there was too much inconsistency in this system.

7. I would imagine that most people would learn to use this system very quickly.

8. I found the system very cumbersome to use.

9. I felt very confident using the system.

10. I needed to learn a lot of things before I could get going with this system.

\subsubsection{Tierney's 7-Minute Accessibility Assessment and App Rating System}

Through interview-administered surveys with both closed and open-ended questions, we asked participants to describe their difficulties while using the app and share their ideas for changes to improve app accessibility and usability. Participants responded to the following three items, adapted from Tierney's app rating system [34]:

1. Please rate your experience completing [name of task or task section], based on ease of use, on a scale of 1 to 5 stars using the following rating key:

- 1 Star: Very Difficult (lowest tolerance for issues)—Unable to complete most tasks independently; Prohibitive confusion or frustration

2 2 Stars: Difficult-Unable to complete a few tasks independently; Significant confusion or frustration

- 3 Stars: Moderate Ease/Difficulty-Able to complete all tasks independently; Moderate confusion or frustration

- 4 Stars: Easy_Able to complete all tasks independently; Infrequent confusion or frustration

○ 5 Stars: Very Easy (highest tolerance for issues) - Able to complete all tasks independently; Minimal to no confusion or frustration

2. While completing [name of task or task section], was there anything of note, that made the process difficult? If yes, please describe the source(s) of difficulty.

3. While completing [name of task or task section], was there anything of note, that you would want to change about the process or app interface to make accomplishing the tasks easier? If yes, please describe what you would like to see changed.

We made minor adaptations to the wording describing Tierney's app rating system to improve the simplicity, clarity, and standardized readability of each item for participants and people unfamiliar with principles of mobile device accessibility.

The 5-star accessibility ratings provide valuable context, when paired with the qualitative feedback gained from targeted questions asked following each task completion. 
The 5-star ratings also serve as an analog to commercial product-review consumer ratings (typically seen in app stores and on sites like Amazon.com).

\subsection{Participant Recruitment and Testing Procedure}

To evaluate the utility of our concurrent mixed methods assessment protocol in generating useful app accessibility and usability information, we recruited a diverse sample of people with disabilities: a third who have dexterity impairments, a third who have visual impairments, and a third who have cognitive impairments. We recruited participants via an emailed recruitment letter, which outlined the study's objectives and participation requirements, and provided basic information about the apps being assessed. Interested respondents were sent a link to an online informed consent form and intake survey that collected information about their demographics and assistive technology usage to access their mobile device.

Before beginning the assessment, participants downloaded the app from the Apple or Google Play store onto their personal mobile device. Participants were then asked to complete a guided series of accessibility testing tasks, adapted from the Tierney assessment model [34]. We asked participants to describe their experience completing the tasks within each app, using the SUS and Tierney assessment measures. We recorded participants' responses in a secure data collection and management tool (REDCap).

To supplement each participant's accessibility and usability assessment, one of the researchers observed the participant's interactions with the app and completed a "significance and impact" assessment, to document unstated but notable accessibility/usability issues experienced during task completion. We combined the observational assessment with the participant's qualitative feedback, overall Tierney " 5 -star" accessibility rating, and total SUS scores for each app.

\section{Results}

\subsection{Participant Characteristics and Assistive Technology Usage}

Table 1 summarizes the nine participants' reported demographic characteristics, collected in the study's initial intake survey. There are equal number of participants across impairment categories with an average age of 41 years old and an average time with impairments at 16 years. There are nearly equal numbers of males and females, and the average annual income is USD 22,000 with a notable range (less than USD 15,000 to between USD $100,000$ and 150,000$)$. This annual income distribution did not notably vary by impairment group. Slightly more than half of respondents use an iOS mobile platform, and the rest use Android mobile operating systems.

Table 1. Participant Demographic Characteristics $(\mathrm{N}=9)$.

\begin{tabular}{cc}
\hline Demographic Variable & Participant Sample Characteristics \\
\hline Impairment Category & 3 Dexterity, 3 Visual, 3 Cognitive \\
\hline Age (years) & Mean: 41; range: 25-64 \\
\hline Time with Impairments (years) & Mean: 16; range: 1-37 \\
\hline Gender & 4 Female, 5 Male \\
\hline Race/Ethnicity & 1 Black, 8 White; 2 Hispanic/Latino \\
\hline Level of Education (years) & Mean = 16; range = 14-18 \\
\hline Annual Income & Mean: USD 22 K; range: <USD 15 K to USD 100-150 K \\
\hline Mobile Platform & 5 iOS, 4 Android
\end{tabular}

Figure 1 summarizes the nine participants' reported usage of assistive technology, accessibility software, and built-in general consumer aids while using mobile devices, also collected in the study's intake survey. Each participant relied upon at least one form of 
technical accommodation to use their mobile phone. The represented technology usage distribution is color-sorted by participant impairment category. Of the eleven assistive technology and general consumer aid options, the most commonly used technologies were Intelligent Personal Assistant (66.6\% of the dexterity group; $100.0 \%$ of the visual group; $33.3 \%$ of the cognitive group) followed by Word Prediction and Autocorrection Software ( $66.6 \%$ of the visual group; $66.6 \%$ of the cognitive group), and Auditory Alerts ( $66.6 \%$ of the visual group; $33.3 \%$ of the cognitive group). The least used assistive technology is Braille Application (33.3\% of the visual group), followed by Accessible Keyboard (66.6\% of the visual group), and Custom Contrast or Color Adjustment (66.6\% of the cognitive group). Of note, participants with dexterity impairments used only two of the eleven technology aids: Assistive Devices/Alternative Access Software (66.6\% of the dexterity group) and Intelligent Personal Assistant (66.6\% of the dexterity group), while participants with visual impairments used nine and participants with cognitive impairments used seven of the eleven listed technology aids.

Assistive Devices / Alternative Access Software

Screen Reader

Braille Application

Accessible Keyboard

Icon, Text, or Screen Magnification

Custom Contrast or Color Adjustment

Word Prediction and Autocorrection Software

Auditory Alerts

Incoming Calls to Headset or Speaker

Intelligent Personal Assistant
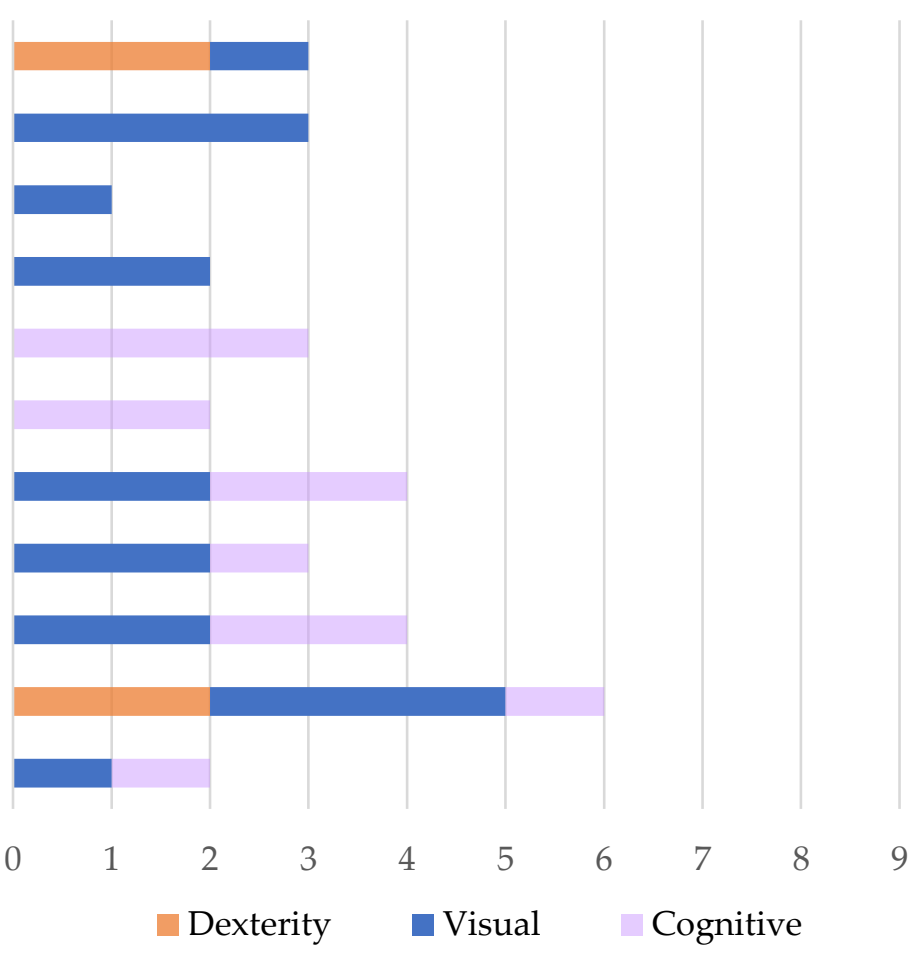

Figure 1. Assistive Technology Usage by Impairment Category $(\mathrm{N}=9)$.

\subsection{Quantitative Results}

\subsubsection{Quantitative SUS Score Results}

Table 2 summarizes the total SUS scores per disability group for all app testing sessions. One-way analysis of variance (ANOVA) was used to compare mean differences between disability groups on SUS scores (0-100) for the task-completion section of each app. Analysis of variance is a common statistical test which examines the mean distributions between three or more unrelated groups to determine if the groups' averages are significantly different from each other. When an ANOVA test results in an alpha below 0.05 , there are significant differences between the averages of the groups. The F statistic is the outcome of the ANOVA paired with the degrees of freedom (df) to determine statistical significance, which is commonly referred to as the $p$ value. $p$ values less than 0.05 are statistically significant (Sig.). Additionally, the number of respondents in each group is reported (N) [40]. 
Table 2. SUS Results-Mean participant SUS Scores (0-100) across three apps, one-way analysis of variance (ANOVA).

\begin{tabular}{cccccccc}
\hline \multirow{2}{*}{ mHealth App } & $\begin{array}{c}\text { Task Completion } \\
\text { Section }\end{array}$ & $\begin{array}{c}\text { Dexterity } \\
\left(\mathbf{N}=\mathbf{3}^{\mathbf{1}}\right.\end{array}$ & $\begin{array}{c}\text { Visual } \\
\mathbf{( N = 3 )}\end{array}$ & $\begin{array}{c}\text { Cognitive } \\
\mathbf{( N = 3 )}\end{array}$ & \multicolumn{2}{c}{ ANOVA Results } \\
\cline { 3 - 8 } & $\mathbf{M}$ & $\mathbf{M}$ & $\mathbf{M}$ & $\mathbf{F}$ & $\mathbf{P}$ & Sig. \\
\hline MyFitnessPal & Explore & 77.5 & 44.2 & 70.0 & 0.853 & 0.480 & $\mathrm{~ns}$ \\
$(\mathrm{~N}=8, \mathrm{df}=7)$ & Core Tasks & 70.0 & 61.7 & 78.3 & 0.812 & 0.495 & $\mathrm{~ns}$ \\
\hline Lose It! & Explore & 83.3 & 31.7 & 81.7 & 14.286 & 0.005 & $*$ \\
$(\mathrm{~N}=9, \mathrm{df}=8)$ & Core Tasks & 89.2 & 47.5 & 82.5 & 3.601 & 0.094 & $\mathrm{~ns}$ \\
\hline FatSecret & Explore & 94.2 & 41.7 & 89.2 & 17.028 & 0.003 & $*$ \\
$(\mathrm{~N}=9, \mathrm{df}=8)$ & Core Tasks & 90.8 & 57.5 & 77.5 & 2.593 & 0.154 & $\mathrm{~ns}$ \\
\hline
\end{tabular}

${ }^{1}$ Dexterity $\mathrm{N}=2$ for MyFitnessPal and $\mathrm{N}=3$ for Lose It! and FatSecret. $\mathrm{N}=$ disability group size, $\mathrm{df}=$ degrees of freedom, $\mathrm{M}=$ Mean, $\mathrm{F}=\mathrm{F}$ statistic, $\mathrm{P}=p$ value, Sig. = statistical significance level, $\mathrm{ns}=$ not significant; ${ }^{*} p<0.05$.

Table 2 illustrates that participants with visual impairments had greater difficulty in completing evaluation tasks for all three apps, compared with other disability groups. These between-group differences were statistically significant for the "Explore" activities with the Lost It! and FatSecret apps. For MyFitnessPal, there were no notable differences in SUS total scores between disability groups while exploring and completing core tasks in the app. For Lose It!, participants with visual impairments reported significantly lower total SUS scores than participants without visual impairments while initially exploring the Lose It! mobile interface $(\mathrm{F}(9)=14.286, p=0.005)$. Similarly, participants with visual impairments reported significantly lower scores than participants with dexterity or cognitive impairments $(\mathrm{F}(9)=17.028, p=0.003)$ while initially exploring the FatSecret app. The overall pattern is clear and consistent across app evaluations: where substantive differences between impairment groups exist, participants with visual impairments report the lowest overall usability scores and the most significant accessibility issues, compared to participants without visual impairments, especially while initially exploring an app interface.

\subsubsection{Quantitative Tierney Rating Results}

Figure 2 presents mean Tierney 5-Star accessibility ratings, representing participants' perceived ease of use for each mHealth app, overall, and for each participant disability group. On average, participants with dexterity impairments reported higher Tierney Accessibility ratings, indicating they experienced the least amount of difficulty (as a group) while using each of the three apps (mean ranges from 4.4 to 4.9 out of 5 across apps). Participants with visual impairments reported the lowest Tierney accessibility ratings, indicating they experienced the most difficulty using the three apps (mean ranges from 2.8 to 3.3 out of 5). Participants with cognitive impairments consistently provided medium-high Tierney accessibility ratings (mean ranges from 4.1 to 4.4 out of 5 ) for each mHealth app.

On average across impairment groups, FatSecret was rated highest for accessibility (4.1), followed by Lose It! (4.0), while MyFitnessPal was rated lowest (3.8) for accessibility. Participants with dexterity impairments rated the Lose It! app highest for accessibility (4.9). Participants with visual impairments and cognitive impairments rated FatSecret highest for accessibility on Tierney's app accessibility rating scale (3.3 and 4.4, respectively).

There are no published studies employing Tierney App Accessibility Ratings. However, Tierney notes the level of associated accessibility with each rating as follows [34]:

- 1 Star: Very Low-Severe accessibility issues and overall inattention to accessibility will prevent some users from using the app.

- $\quad 2$ Stars: Low-Accessibility issues will prevent some users from completing core tasks in the app, due to poor overall accessibility quality.

- 3 Stars: Moderate-Accessibility issues may prevent some users from completing noncore tasks. Users may experience significant annoyances and confusion, due to lack of attention to detail across the application. 
- 4 Stars: High-Some minor impact issues may exist, but no accessibility issues prevent user from completing core tasks, despite annoyances.

- 5 Stars: Very High-Low incidence of issues. Some minor impact issues may exist, but no accessibility issues prevent user from completing core tasks.

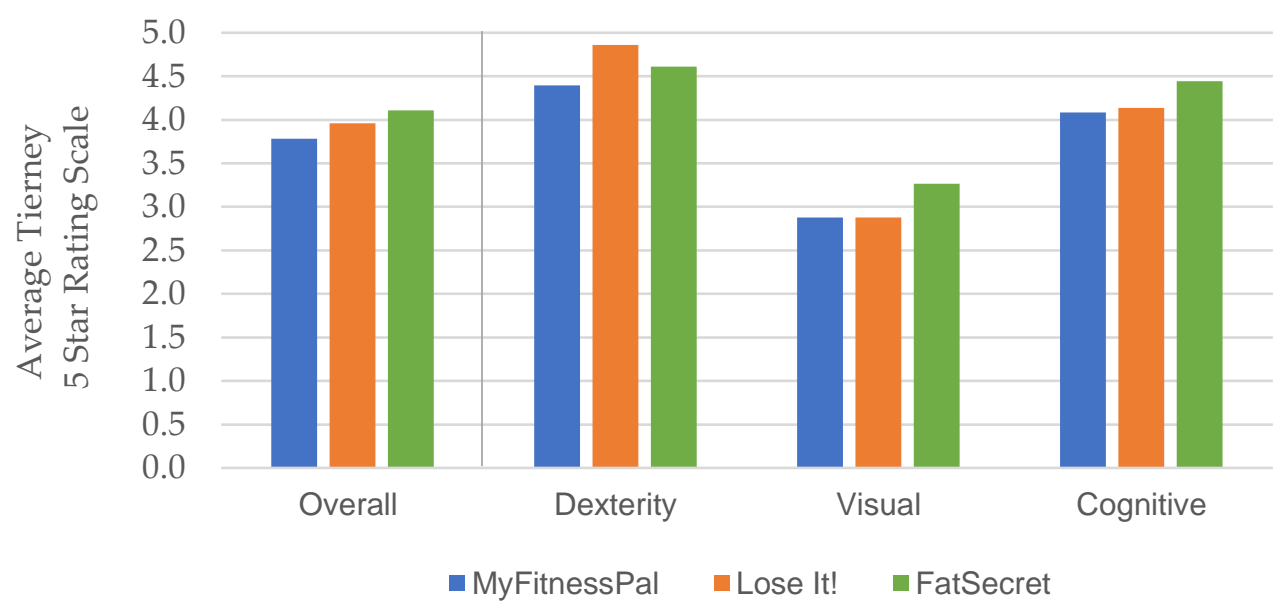

Figure 2. Mean participant Tierney ratings (1-5) both overall and by disability category per mHealth app.

\subsection{Qualitative Results}

While completing the "Explore" and "Core Tasks" sections of our app accessibility testing protocol, assessing MyFitnessPal, Lose It!, and FatSecret, the following themes emerged:

Participants with dexterity impairments experienced significant difficulties, especially in instances where functional elements were either not available or more cumbersome for them to use. Difficulties occurred when built-in voice controlled mobile accessibility features, utilizing vocally prompted commands or numerical grid overlays, could not successfully manipulate standard app elements, or when clickable app elements were unresponsive to a user's assistive device inputs.

One participant with dexterity impairments using iOS Voice Control (a built-in accessibility feature that enables Apple phone use through voice commands) was unable to easily navigate within MyFitnessPal because the accessibility feature's overlay could not consistently access clickable buttons and fields within the app interface [41]. Another participant with dexterity impairments, using a Bluetooth-paired GlassOuse assistive device (a wearable gyroscope-based system worn like glasses to enable cursor control using head movements and clicking action using a connected bite-switch), was unable to control the MyFitnessPal app interface, because many of the entry fields were unresponsive or overly sensitive to the device inputs [42].

Participants with visual impairments experienced the most usability and accessibility difficulties, especially while exploring each app, due to frequently encountered instances of unlabeled buttons, icons, navigational elements, screen headings, and entry-field labels within each app. These participants, using a screen-reader to access their mobile devices, often got stuck, lost, discouraged, and confused while using each app, and they often required assistance to gain sufficient context to locate and use standard functions during and between tasks.

Alt Text was inconsistently integrated throughout each app, and participants with visual impairments using screen-readers were unable to read or access wrapping menus, graphic data, and most media on MyFitnessPal's home screen (e.g., blog posts, workout videos, and recipes). Graphical representations of data in Lose It! were also not accessible to participants with visual impairments using screen-readers.

Participants with cognitive impairments experienced moderate difficulties or confusion while completing sub-portions of each task completion section per app, but they tended to have higher tolerances for each experienced issue and were consistently able 
to complete each task in the protocol without assistance. These participants struggled to consistently intuit the purpose of certain buttons with unclear text-labeling and sometimes struggled to read elements with low-contrast text-to-background labels and icons in each app. They frequently requested guidance, commenting that an info tab or hyperlink to instructions for how to use important app features would be helpful to them.

\section{Discussion}

As mHealth app usage and development continues to grow, it is increasingly important that apps be accessible and usable for the widest range of human abilities. Harrison et al. conducted a review of mobile usability models and notes that usability is often measured by three core attributes: effectiveness, efficiency and satisfaction, though other important attributes, such as cognitive load and utility tend to be disregarded, despite their likely influence on the level of a mobile application's usability [43]. This study introduced a more comprehensive usability model designed to address the limitations of existing models when applied to mobile devices [43]. This multifaceted usability framework can be applied in parallel with publicly available accessibility guidelines to understand the range of user needs that must be considered when developing, assessing, and selecting suitable mHealth applications.

Various sets of accessibility guidelines have been established, such as the World Wide Web Consortium (W3C) Web Content Accessibility Guidelines (WCAG) 2.0 and the User Agent Accessibility Guidelines (UAAG) 2.0 published by the W3C Web Accessibility Initiative (WAI) [44-46]. Notably, the two leading multinational technology companies that design and develop the majority of the world's mobile apps, Google LLC and Apple Inc, have published extensive amounts of information for developers on how to develop, improve, and test for accessibility on Android and Apple mobile applications [47-52]. Both Google and Apple have also created and enabled the integration of compatible mobile accessibility features that are now either built-in or publicly available for all Android and iOS mobile device users [53,54]. These native consumer software accessibility features aim to support mobile device users with visual, mobility/dexterity, cognitive, and hearing impairments. However, as noted in our results and in the results of other larger-scale studies evaluating the accessibility and usability of mobile apps, accessibility issues remain prevalent in commercial mobile apps, indicating the need for informed support for both app consumers with disabilities and mobile app developers [55-57].

A study completed by Yan et al. assessed the level of accessibility in 479 Android mobile apps using an automated accessibility evaluation tool and found a pervasive incidence of accessibility issues across the sample of evaluated mobile apps, primarily caused by "missing element descriptions, low text color contrast, lacking element focus," insufficient spacing between elements, and "less than minimum sizes of text and elements" [55]. Eler et al. also explored automated test generation and employed a mobile accessibility testing tool to evaluate 73 apps for accessibility issues affecting users with visual impairment [56]. This study reveals that both manual accessibility testing methods and the automated accessibility checker detected numerous but differing accessibility issues in the sample of evaluated mobile apps, even when mobile accessibility aids were enabled during testing [56]. These results highlight the value of both automated and manual mobile app accessibility evaluations as complimentary methods.

Results obtained through automated accessibility evaluation tools align with insight gleaned from our small-scale, in-person app accessibility testing results, as both discovered shared themes regarding the frequency and nature of common mobile app accessibility issues. Given that our protocol detected accessibility issues experienced by people with disabilities in real time, we were able to not only detect accessibility non-conformance, but also observe and note the impact that the accessibility issues made on the app user's ability to complete tasks for intended use cases within the apps we evaluated.

Aguado-Delgado et al. presented a comprehensive approach for evaluating the accessibility and usability of mobile apps informed by W3C recommendations and usability 
heuristic evaluation methods [57]. Similar to our protocol design, app accessibility evaluations in this study involved observing participants' completion of guided tasks within the mobile application's relevant scenarios in real time while documenting observed and reported accessibility issues along with each issue's severity and impact on the participant's successful completion of tasks within the app [57]. The results of this work conclude that a comprehensive evaluation of mobile app accessibility and usability, using a similar systematic procedure informed by prior art, can be completed by non-expert evaluators "in a short period of time" without the use of automated accessibility evaluation tools [57].

Our pilot protocol enables an informed and practical approach for assessing the accessibility and usability of mHealth apps with participants with disabilities to generate actionable insight for mHealth app consumers with similar impairments to assist their selection of optimal mHealth apps. While our small-scale quantitative analyses provide a basis for comparison of accessibility and usability for each evaluated app by disability group, our participants' qualitative feedback provides valuable information to help consumers select the mHealth app that may be best suited to their needs and abilities. This systematically collected qualitative data also identifies specific areas within each evaluated app that require attention and effort from app developers towards accessibility and usability improvements.

If any aspect or function within a weight management app (e.g., login, navigational elements, meal diary entry, exercise logging, graphical data) is not accessible, a user is likely to become frustrated or discouraged, increasing their likelihood of abandoning the app and not benefitting from the support it was designed to offer. Consequently, lacking or inconsistent accessibility throughout an mHealth application risks reinforcing the preexisting disparity between people with disabilities and non-disabled populations in accessing publicly available health and wellness resources. Our methodically acquired qualitative results inform guidance to app developers on how to specifically improve each app's accessibility and usability for participants with dexterity, visual, and cognitive impairments, as well as for general app consumers.

\subsection{Opportunities for Improved Mobile App Accessibility:}

To address the needs and use-cases of people with dexterity impairments who use built-in Voice Control/Voice Access accessibility features to control their mobile devices, we recommend that app developers design and test app production interfaces with accessibility feature overlay constraints in mind, to ensure that key functional buttons and navigational elements are accessible to app users using built-in accessibility features $[46,47,49,51]$. We also suggest that app developers enable the options to manually enter values via voice entry or a standard on-screen keyboard, rather than requiring certain data-entries to be submitted solely via a scroll-wheel. For iOS Voice Control users, entry fields requiring typing could be simplified by enabling users to enter data via spoken entries. To address the needs of people with dexterity impairments who use switch-control devices to operate their mobile devices, we recommend that developers iteratively test and refine functional element and field sensitivities within the app to translate assistive device enabled user-inputs more accurately $[46,49,51]$.

To address the needs of people with visual impairments using screen-readers, we recommend that developers incorporate consistently integrated Alt Text for all functions, navigational elements, graphical representations, and data-entry fields [44,46,48,50,52]. We specifically recommend that app developers provide clearly labeled, and consistently located "back" and "next" buttons within each entry field and sub-app screen to provide visually impaired users with sufficient context for successful app navigation and use. To make app use more inclusive for participants with visual impairments, developers will also need to incorporate sufficient Alt Text in visual data representations (e.g., graph and chart elements) to enable app users utilizing a screen-reader to read, interact with, and gain equal benefits from graphical app features [46,50,52]. 
To improve ease of use and reduce preventable confusion or difficulty for app users with cognitive impairments, along with general consumers, we recommend that developers focus on incorporating clear and consistent labels for buttons, icons, and fields along with high text-to-background contrast when text is used to explain an element's function $[43,46,47,50]$. Based on feedback from participants with cognitive impairments, we also suggest that app designers enable the capacity for users to show/hide login credential entries while typing, so that users can temporarily note what they have already typed during the data-entry process, rather than having to restart an entry each time they lose track of their entry field completion progress. Providing these options would likely benefit general consumers in increasing app ease of use $[43,47,50]$.

Inconsistency in mobile interface functions, labels, displays, and entry-field types in an app can cause significant confusion and uncertainty among all app users, regardless of impairment type. To maximize usability during app use for consumers with various ability levels, developers must design apps as cohesive systems with unified structure, process-flows, and themes.

\subsection{Limitations}

Given the small participant sample size, typical of usability testing studies, results obtained through our quantitative analyses have low statistical power. Additionally, the small sample of participants is diverse both in demographic and impairment characteristics. This participant variance influences the consistency of both our quantitative and qualitative findings. Measured results and SUS score differences between impairment groups per mHealth app are not intended to infer quantitative trends, but more-so, are meant to provide objectively measured context when paired with richer, subjective qualitative analyses of participant experiences.

\subsection{Future Work}

As noted in the introduction, the objective of this study was to develop and test a protocol that can be used to systematically gather input from people with disabilities on app accessibility and usability, in order share ratings and insights with other prospective users with disabilities. To this end, we are building an app curation website, www.theappfactory. org, where the app testing results will be published. The website will have three purposes:

1. To provide app users with disabilities clear ratings and information on accessibility issues they may encounter while using each evaluated app, based on their disability or the assistive technology they may use to access their mobile devices. This information is meant to help app users make informed decisions when selecting an mHealth app to meet their needs.

2. To invite and enable app users with disabilities to provide their own accessibility ratings and "consumer reviews" for selected mHealth apps.

3. To make recommendations to developers on steps they can take to improve their app's accessibility and usability for people with disabilities.

Based on a review of the current state, opportunities and challenges, and future needs of mobile health for people with disabilities, we plan to refine and apply our app accessibility and usability testing methodology towards the evaluation of a diverse set of mHealth/mRehab apps focused on supporting various aspects of health and wellness [4,58,59]. Informed by results from a recent survey of user needs and preferences for mHealth apps by people with disabilities (physical, cognitive, sensory, emotional/psychological, and speech), our future work will focus on testing the accessibility and usability of the following mHealth/mRehab app categories [60].

- Apps that track and support individualized goals and health habits;

- Apps that track multiple areas of health in one app;

- Clinical portal apps that enable personal health information management, appointment scheduling, access to lab results and vital sign tracking, and correspondence with healthcare providers (e.g., MyChart); 
- $\quad$ Apps that support stress, PTSD, and mental health management;

- Apps that track and support healthy sleep patterns;

- Apps that support diabetes management via blood pressure, blood sugar, and heart rate monitoring;

- Sensor-enhanced activity monitoring apps that support remote physical rehabilitation;

- Exercise apps for wheelchair users.

These app categories are prioritized based on the reported mHealth apps most used or deemed valuable and necessary by survey respondents with disabilities [60]. Though we initially reasoned that apps supporting chronic health condition management would be important to include, survey resulted indicated that symptom and disease management apps were the least commonly used by respondents with disabilities [60]. In the next phase of this project, we will focus on the first two bullets listed above and aim to evaluate apps that track multiple areas of health to support individualized goals and health habits. We are looking forward to testing the accessibility and usability of this next identified collection of popular mHealth apps by people with disabilities.

\section{Conclusions}

Study results inform guidance for consumers with disabilities in choosing mHealth apps that meet their needs, and findings reveal common accessibility issues to inform app developers of key inclusive design requirements. Accessibility issues in popular mHealth apps for weight management hinder people with disabilities from successfully using and fully benefiting from these apps' support in reaching diet, weight, and exercise goals. Maintaining a diet and exercise regimen for people with disabilities is more important now than ever during the COVID-19 pandemic, as many people have been unable to get out and enjoy their normal workout routines or easily access healthy food options. Apps continue to be developed at a rapid pace, and currently, over 2.8 million apps are available on Google Play while over 1.9 million apps are available in the Apple App Store [61]. Fulfilling inclusive design requirements often enables more positive usability experiences for both impaired and general consumers, and app developers need technical instruction to ensure their mobile interfaces are designed for accessibility to enable use by the widest range of human abilities. Moving forward, our evaluation protocol will be used to assess additional mHealth apps and populate an app "curation" website to assist consumers in locating accessible and usable mHealth apps and to inform developers of opportunities for improved mobile app accessibility.

Author Contributions: Conceptualization, M.J., E.R. and B.L.; methodology, M.J., E.R. and B.L.; validation, E.R. and B.L.; formal analysis, E.R. and R.A.; investigation, E.R. and B.L.; data curation, E.R. and B.L.; writing — original draft preparation, E.R., B.L. and R.A.; writing—review and editing, M.J., E.R., B.L. and R.A.; project administration, E.R. and B.L. All authors have read and agreed to the published version of the manuscript.

Funding: This research was conducted by the App Factory Development and Rehabilitation Research Project (App Factory DRRP), which is funded by the National Institute on Disability, Independent Living and Rehabilitation Research (NIDILRR) of the U.S. Department of Health and Human Services, Grant number 90DPHF004. The opinions contained in this document are those of the grantee and do not necessarily reflect those of the U.S. Department of Health and Human Services or NIDILRR.

Institutional Review Board Statement: The study was conducted according to the guidelines of the Declaration of Helsinki and approved by the Institutional Review Board (or Ethics Committee) of Shepherd Center (protocol code 803 and approved on 19 December 2019).

Informed Consent Statement: Informed consent was obtained from all subjects involved in the study.

Data Availability Statement: The data presented in this study are available upon request from the corresponding author at erin.radcliffe@shepherd.org. The data are not publicly available due to data privacy ethics. 
Conflicts of Interest: The authors declare no conflict of interest. The funders had no role in the design of the study; in the collection, analyses, or interpretation of data; in the writing of the manuscript, or in the decision to publish the results.

\section{References}

1. Kinne, S.; Patrick, D.L.; Lochner, D.D. Prevalence of secondary conditions among people with disabilities. Am. J. Public Health 2004, 94, 443-445. [CrossRef] [PubMed]

2. Krahn, G.L.; Reyes, M.; Fox, M. Chronic conditions and disability: Toward a conceptual model for national policy and practice considerations. Disabil. Health J. 2014, 7, 13-18. [CrossRef] [PubMed]

3. Iezzoni, L.I. Eliminating health and health care disparities among the growing population of people with disabilities. Health Aff. 2011, 30, 1947-1954. [CrossRef] [PubMed]

4. Jones, M.; Morris, J.; Deruyter, F. Mobile healthcare and people with disabilities: Current state and future needs. Int. J. Environ. Res. Public Health 2018, 15, 515. [CrossRef]

5. Ballantyne, M.; Archit, J.; Anna, J.; Hawker, J.S.; El-Glaly, Y.N. Study of Accessibility Guidelines of Mobile Applications. In Proceedings of the 17th International Conference on Mobile and Ubiquitous Multimedia (MUM 2018), Cairo, Egypt, 25-28 November 2018; Association for Computing Machinery: New York, NY, USA, 2018; pp. 305-315.

6. Siebra, C.; Anjos, M.; Florentin, F.; Gouveia, T.; Filho, A.; Correia, W.; Penha, M.; Silva, F.; Santos, A. Accessibility Devices for Mobile Interfaces Extensions: A Survey. In Proceedings of the 17th International Conference on Human-Computer Interaction with Mobile Devices and Services Adjunct (MobileHCI '15), Copenhagen, Denmark, 24 August 2015; Association for Computing Machinery: New York, NY, USA, 2015; pp. 644-651.

7. Damaceno, R.J.P.; Braga, J.C.; Mena-Chalco, J.P. Mobile device accessibility for the visually impaired: Problems mapping and recommendations. Univ. Access Inf. Soc. 2018, 17, 421-435. [CrossRef]

8. Alajarmeh, N. The extent of mobile accessibility coverage in WCAG 2.1: Sufficiency of success criteria and appropriateness of relevant conformance levels pertaining to accessibility problems encountered by users who are visually impaired. Univ. Access Inf. Soc 2021, 1-26. [CrossRef]

9. Dixon-Ibarra, A.; Horner-Johnson, W. Disability status as an antecedent to chronic conditions: National health interview survey, 2006-2012. Prev. Chronic. Dis. 2014, 11, 1-8. [CrossRef]

10. Morris, J.; Jones, M.; Sweatman, M. Wireless technology use by people with disabilities: A national survey. J. Technol. Pers. Disabil. 2016, 101-113.

11. Dombrowski, S.U.; Sniehotta, F.F.; Avenell, A.; Johnston, M.; MacLennan, G.; Araújo-Soares, V. Identifying active ingredients in complex behavioural interventions for obese adults with obesity-related co-morbidities or additional risk factors for comorbidities: A systematic review. Health Psychol. Rev. 2012, 6, 7-32. [CrossRef]

12. Michie, S.; Ashford, S.; Sniehotta, F.F.; Dombrowski, S.U.; Bishop, A.; French, D.P. A refined taxonomy of behaviour change techniques to help people change their physical activity and healthy eating behaviours: The CALO-RE taxonomy. Psychol. Health 2011, 26, 1479-1498. [CrossRef]

13. Coughlin, S.S.; Whitehead, M.; Sheats, J.Q.; Mastromonico, J.; Hardy, D.; Smith, S.A. Smartphone Applications for Promoting Healthy Diet and Nutrition: A Literature Review. Jacobs J. Food Nutr. 2015, 2, 1-14.

14. Coughlin, S.S.; Whitehead, M.; Sheats, J.Q.; Mastromonico, J.; Smith, S. A Review of Smartphone Applications for Promoting Physical Activity. Jacobs J. Community Med. 2016, 2, 1-22.

15. Jake-Schoffman, D.E.; Turner-McGrievy, G.M.; Jones, S.J.; Wilcox, S. Mobile apps for pediatric obesity prevention and treatment, healthy eating, and physical activity promotion: Just fun and games? TBM 2013, 3, 320-325.

16. Barlow, S.E. The Expert Committee, Expert committee recommendations regarding the preventions, assessment, an treatment of child and adolescent overweight and obesity: Summary report. Pediatrics 2007, 120, S164-S192. [CrossRef]

17. Bondaronek, P.; Alkhaldi, G.; Slee, A.; Hamilton, F.L.; Murray, E. Quality of Publicly Available Physical Activity Apps: Review and Content Analysis. JMIR Mhealth Uhealth. 2018, 6, 1-15. [CrossRef] [PubMed]

18. Bardus, M.; van Beurden, S.; Smith, J.; Abraham, C. A review and content analysis of engagement, function, aesthetics, information quality, and change techniques in the most popular commercial apps for weight management. Int. J. Behav. Nutr. Phys. Act. 2016, 13, 1-9. [CrossRef] [PubMed]

19. My Fitness Pal. Available online: https:/ / www.myfitnesspal.com (accessed on 2 September 2020).

20. Lose It! Available online: https:/ / www.loseit.com (accessed on 20 December 2020).

21. Fat Secret Calorie Counter App. Available online: http://www.FatSecret.com (accessed on 2 September 2020).

22. Noom. Available online: https: / / www.noom.com (accessed on 23 March 2021).

23. WW (Weight Watchers). Available online: https://www.weightwatchers.com (accessed on 23 March 2021).

24. MyPlate. Available online: https:/ / www.livestrong.com/myplate (accessed on 23 March 2021).

25. VA MOVE! Coach. Available online: https://www.move.va.gov/MOVE/movecoach.asp (accessed on 23 March 2021).

26. MyNetDiary. Available online: https:/ / www.mynetdiary.com (accessed on 23 March 2021).

27. My Diet Diary. Available online: https:/ / www.mydietdiary.co.uk (accessed on 23 March 2021).

28. SparkPeople. Available online: https:/ / www.sparkpeople.com (accessed on 23 March 2021). 
29. Wharton, C.M.; Johnston, C.S.; Cunningham, B.K.; Sterner, D. Dietary self-monitoring, but not dietary quality, improves with use of smartphone app technology in an 8-week weight loss trial. J. Nutr. Educ. Behav. 2014, 46, 440-444. [CrossRef]

30. Laing, B.Y.; Mangione, C.M.; Tseng, C.H.; Leng, M.; Vaisberg, E.; Mahida, M.; Bholat, M.; Glazier, E.; Morisky, D.; Bell, D. Effectiveness of a smartphone application for weight loss compared with usual care in overweight primary care patients: A randomized, controlled trial. Ann. Intern. Med. 2014, 161, S5-S12. [CrossRef]

31. Serrano, K.J.; Yu, M.; Coa, K.I.; Collins, L.M.; Atienza, A.A. Mining health app data to find more and less successful weight loss subgroups. J. Med. Internet Res. 2016, 18, 1-23. [CrossRef]

32. Turner-McGrievy, G.M.; Beets, M.W.; Moore, J.B.; Kaczynski, A.T.; Barr-Anderson, D.J.; Tate, D.F. Comparison of traditional versus mobile app self-monitoring of physical activity and dietary intake among overweight adults participating in an mHealth weight loss program. J. Am. Med. Inform. Assoc. 2013, 20, 513-518. [CrossRef]

33. Jake-Schoffman, D.E.; Silfee, V.J.; Waring, M.E.; Boudreaux, E.; Sadasivam, R.; Mullen, S.P.; Carey, J.L.; Hayes, R.B.; Ding, E.Y.; Bennett, G.G.; et al. Methods for evaluating the content, usability, and efficacy of commercial mobile health apps. JMIR Mhealth Uhealth 2017, 5, 1-12. [CrossRef] [PubMed]

34. Tierney, A. The 7-Minute Accessibility Assessment and App Rating System. In Proceedings of the 32nd Annual CSUN Assistive Technology Conference, San Diego, CA, USA, 2 March 2017; Available online: https:/ /www.slideshare.net/aidantierney/the-7minute-accessibility-assessment-and-app-rating-system (accessed on 19 September 2019).

35. Brooke, J. SUS: A retrospective. J. Usability Stud. 2013, 8, 29-40.

36. System Usability Scale (SUS): A Quick-and-Dirty Usability Scale. Available online: https://hell.meiert.org/core/pdf/sus.pdf (accessed on 2 September 2020).

37. Finstad, K. The system usability scale and non-native English speakers. J. Usability Stud. 2006, 1, 185-188.

38. Bangor, A.; Kortum, P.T.; Miller, J.T. An empirical evaluation of the system usability scale. Int. J. Hum. Comput Interact. 2008, 24, 574-594. [CrossRef]

39. Lewis, J.R. Psychometric evaluation of the PSSUQ using data from five years of usability studies. Int. J. Hum. Comput. Interact. 2011, 14, 463-488.

40. Frankfort-Nachmias, C.; Leon-Guerrero, A. Social Statistics for a Diverse Society, 8th ed.; Sage Publication: Thousand Oaks, CA, USA, 2018; pp. 303-324.

41. Apple. Accessibility-Mobility. Available online: https:/ /www.apple.com/accessibility/mobility/ (accessed on 6 January 2021).

42. GlassOuse Assistive Device. Available online: https://glassouse.com/ (accessed on 6 January 2021).

43. Harrison, R.; Flood, D.; Duce, D. Usability of mobile applications: Literature review and rationale for a new usability model. J. Interact. Sci. 2013, 1, 1-16. [CrossRef]

44. The World Wide Web Consortium (W3C). Web Content Accessibility Guidelines (WCAG) 2.0. Available online: https://www.w3 .org/TR/WCAG20/ (accessed on 26 March 2021).

45. The World Wide Web Consortium (W3C). Mobile Accessibility Examples from UAAG 2.0 Reference. Available online: https: / /www.w3.org/TR/2015/NOTE-UAAG20-Reference-20151215/mobile.html (accessed on 26 March 2021).

46. The World Wide Web Consortium (W3C). Mobile Accessibility at W3C. Available online: https://www.w3.org/WAI/standardsguidelines/mobile/ (accessed on 26 March 2021).

47. Google, LLC. Principles for improving app accessibility. Available online: https://developer.android.com/guide/topics/ui/ accessibility / principles (accessed on 26 March 2021).

48. Google, LLC. Make apps more accessible. Available online: https://developer.android.com/guide/topics/ui/accessibility/apps (accessed on 26 March 2021).

49. Google, LLC. Accessibility Testing Checklist. Available online: https://stuff.mit.edu/afs/sipb/project/android/docs/tools / testing/testing_accessibility.html (accessed on 26 March 2021).

50. Apple, Inc. Human Interface Guidelines. Available online: https://developer.apple.com/design/human-interface-guidelines/ accessibility / overview / best-practices / (accessed on 26 March 2021).

51. Apple, Inc. Testing for Accessibility on OS X. Available online: https://developer.apple.com/library/archive/documentation/ Accessibility/Conceptual/AccessibilityMacOSX/OSXAXTestingApps.html (accessed on 26 March 2021).

52. AppleVis. Information for Developers on how to Build Accessible iOS, iPadOS, Mac, Apple Watch, and Apple TV Apps. Available online: https: / / www.applevis.com/information-developers-how-build-accessible-ios-ipados-mac-apple-watch-and-appletv-apps (accessed on 26 March 2021).

53. Apple. Accessibility. Available online: https:/ / www.apple.com/accessibility/ (accessed on 26 March 2021).

54. Andorid. Accessibility. Available online: https:/ / www.android.com/accessibility / (accessed on 26 March 2021).

55. Yan, S.; Ramachandran, P. The Current Status of Accessibility in Mobile Apps. ACM Trans. Access. Comput. 2019, $12,1-31$. [CrossRef]

56. Eler, M.M.; Rojas, J.M.; Ge, Y.; Fraser, G. Automated Accessibility Testing of Mobile Apps. In Proceedings of the IEEE 11th International Conference on Software Testing, Verification and Validation (ICST), Västerås, Sweden, 9-13 April 2018 ; pp. 116-126.

57. Aguado-Delgado, J.; Castillo-Martinez, A.; Gutierrez-Martinez, J.M.; Hilera, J.R.; de-Marcos, L. Accessibility and usability evaluation techniques for mobile applications: A case study on industrial helping application. Initiatives 2017, 5, 6. 
58. DeRuyter, F.; Jones, M.; Morris, J. Mobile Health and Mobile Rehabilitation for People with Disabilities: Current State, Challenges and Opportunities. In Computers Helping People with Special Needs; Miesenberger, K., Manduchi, R., Covarrubias Rodriguez, M., Peňáz, P., Eds.; Lecture Notes in Computer Science; Springer International Publishing: New York, NY, USA, 2020; Volume 12377, pp. 219-223.

59. World Health Organization. WHO Guideline: Recommendations on Digital Interventions for Health System Strengthening; World Health Organization: Geneva, Switzerland, 2019.

60. Lippincott, B.; Thompson, N.; Morris, J.; Jones, M.; DeRuyter, F. Survey of User Needs: Mobile Apps for mHealth and People with Disabilities. In Computers Helping People with Special Needs; Miesenberger, K., Manduchi, R., Covarrubias Rodriguez, M., Peňáz, P., Eds.; Lecture Notes in Computer Science; Springer International Publishing: New York, NY, USA, 2020; Volume 12377, pp. 266-273.

61. Biggest App Stores in the World 2020. Available online: www.statista.com/statistics/276623/number-of-apps-available-inleading-app-stores / (accessed on 24 November 2020). 